Supporting Information

\title{
New water vapor barrier film based on lamellar
}

\section{aliphatic-monoamine-bridged polysilsesquioxane}

Cong Zhang, ${ }^{, \perp}$ Ce Zhang, ${ }^{t,}{ }^{\perp}$ Ruimin Ding, ${ }^{* *}$ Xinmin Cui, ${ }^{,{ }^{\perp}}$ Jing Wang, ${ }^{t^{\perp}}$ Qinghua Zhang ${ }^{s, *}$ and Yao Xu ${ }^{\text {*** }}$

${ }^{\dagger}$ Institute of Coal Chemistry, Chinese Academy of Sciences, Taiyuan, 030001, China.

${ }^{\ddagger}$ State Key Laboratory of Transient Optics and Photonics, Xi'an Institute of Optics and Precision Mechanics, Chinese Academy of Sciences, Xi'an, 710119, China

${ }^{\S}$ Chengdu Fine Optical Engineering Research Center, Chengdu, Sichuan, 610041, China

${ }^{\perp}$ University of Chinese Academy of Sciences, Beijing, 100049, China.

\section{Corresponding Authors}

*E-mail: xuyao@opt.ac.cn

*E-mail: $\underline{\text { dingrm@sxicc.ac.cn }}$

*E-mail: zhangqh506@163.com 

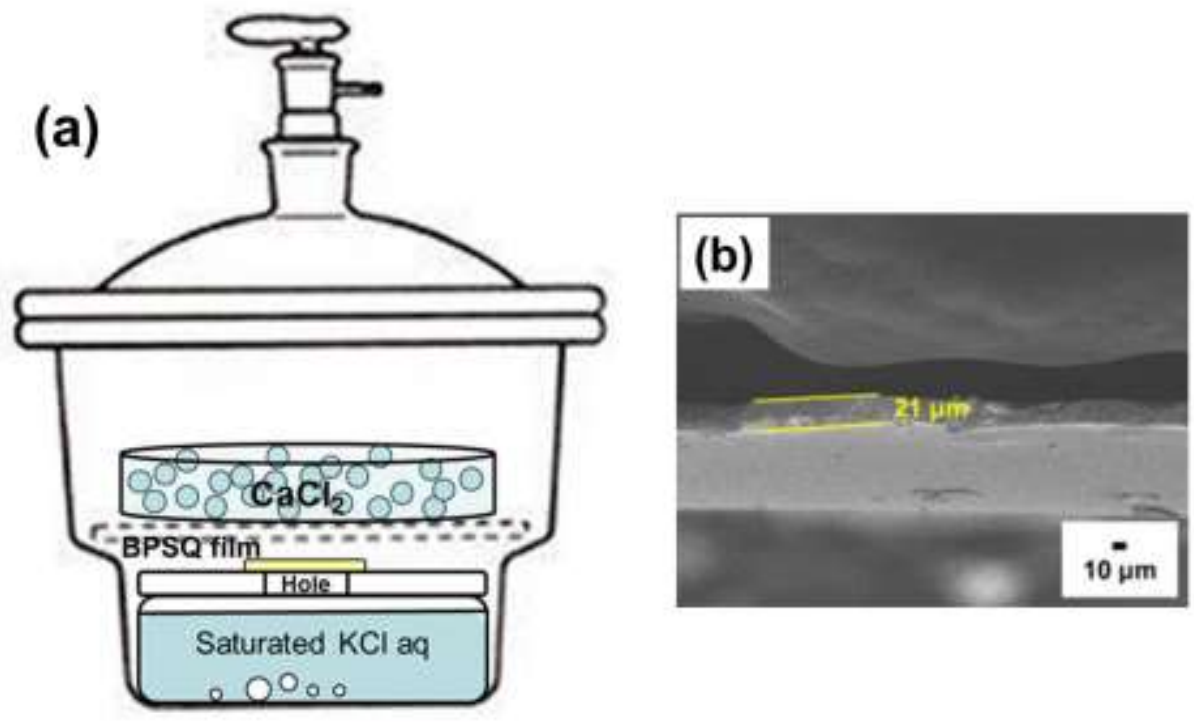

Figure S1 (a). The diagram designed for WVTR test, (b) cross-section of a stand-free HG-BPSQ film for WVTR test.
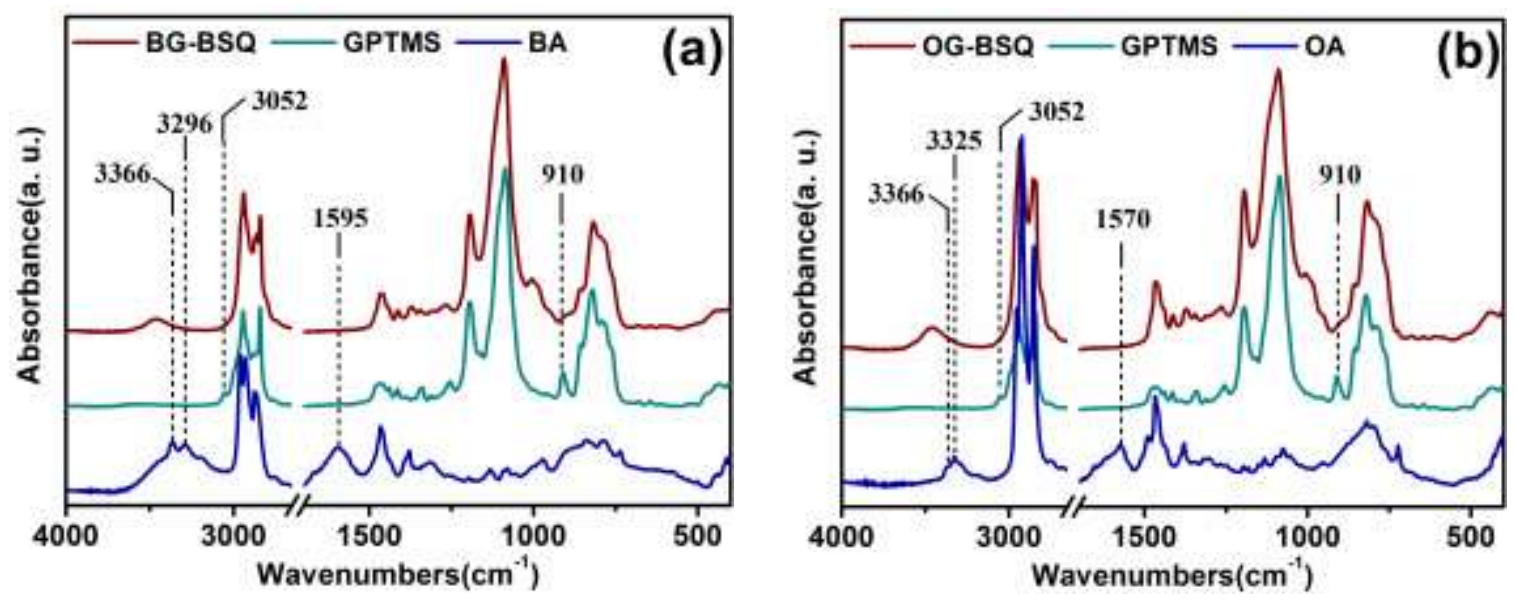

Figure S2. (a) FT-IR spectra of BA, GPTMS and BG-BSQ, and (b) FT-IR spectra of OA, GPTMS and OG-BSQ. 

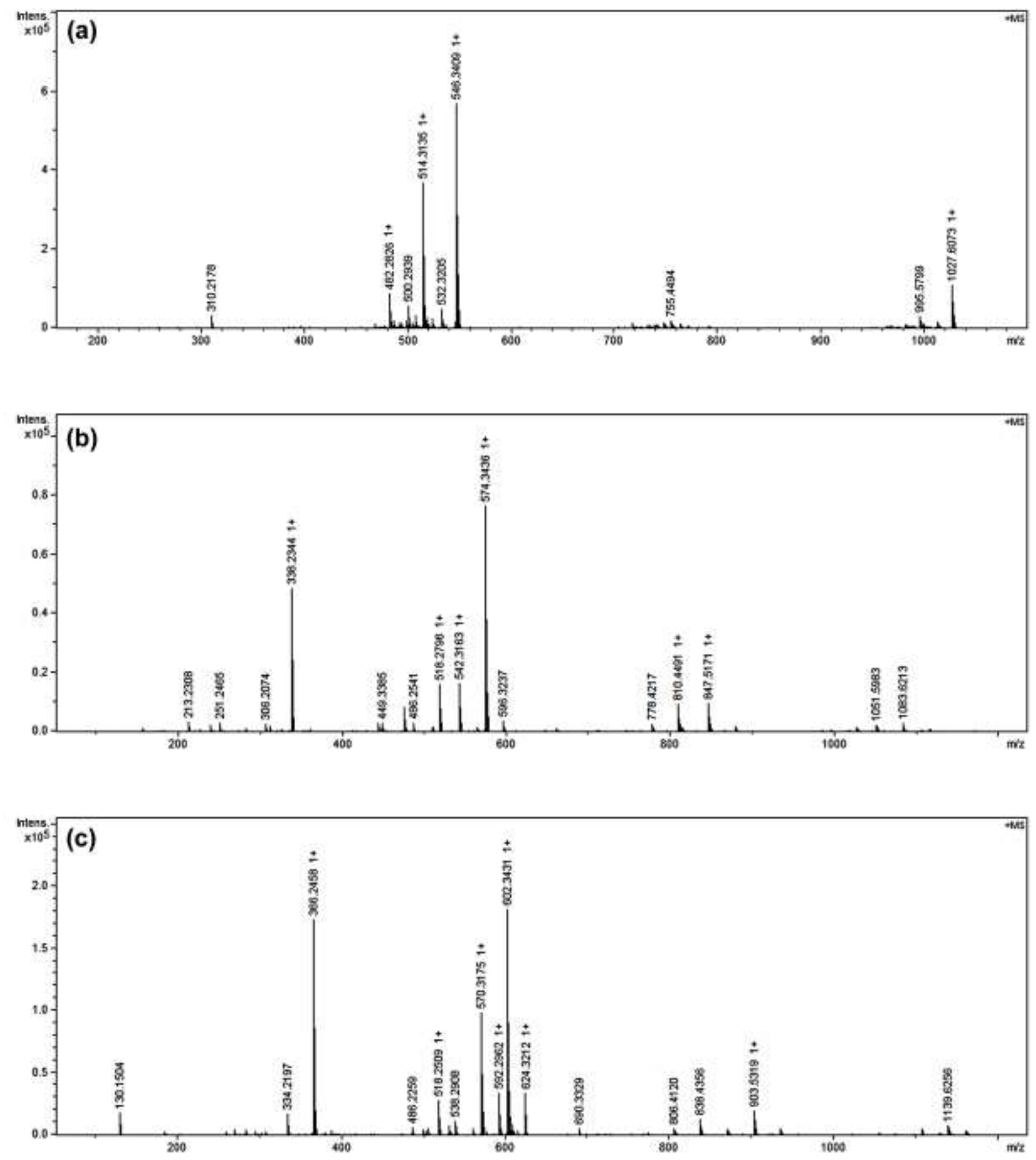

Figure S3. Mass spectra of (a) BG-BSQ, (b) HG-BSQ and (c) OG-BSQ. 
Table S1. The mass-to-charge ratio $(\mathrm{m} / \mathrm{z})$ and its corresponding fragment of the molecular ion peak of BG- BSQ.

\begin{tabular}{|c|c|}
\hline $\mathrm{m} / \mathrm{z}$ & Fragment \\
\hline 310.2178 & {$[\mathrm{M}+\mathrm{H}]^{+}-\mathrm{C}_{9} \mathrm{H}_{20} \mathrm{O}_{5} \mathrm{Si}$} \\
\hline $482.28261+$ & {$[\mathrm{M}+\mathrm{H}]^{+}-2 \mathrm{CH}_{3} \mathrm{OH}$} \\
\hline 500.2939 & {$[\mathrm{M}+\mathrm{H}]^{+}-\mathrm{CH}_{3} \mathrm{OH}-\mathrm{CH}_{2}$} \\
\hline $514.31351+$ & {$[\mathrm{M}+\mathrm{H}]^{+}-\mathrm{CH} 3 \mathrm{OH}$} \\
\hline $546.34091+$ & {$[\mathrm{M}+\mathrm{H}]^{+}$} \\
\hline
\end{tabular}

Table S2. The mass-to-charge ratio $(\mathrm{m} / \mathrm{z})$ and its corresponding fragment of the molecular ion peak of HG- BSQ.

\begin{tabular}{|c|c|}
\hline $\mathrm{m} / \mathrm{z}$ & Fragment \\
\hline 213.2308 & $\mathrm{M}-\mathrm{C}_{9} \mathrm{H}_{20} \mathrm{O}_{5} \mathrm{Si}-3 \mathrm{CH}_{3} \mathrm{OH}-\mathrm{C}_{2} \mathrm{H}_{4}$ \\
\hline 251.2465 & $\mathrm{M}-\mathrm{C}_{9} \mathrm{H}_{20} \mathrm{O}_{5} \mathrm{Si}-\mathrm{C}_{6} \mathrm{H}_{13}$ \\
\hline 306.2074 & $\mathrm{M}-\mathrm{C}_{9} \mathrm{H}_{20} \mathrm{O}_{5} \mathrm{Si}-\mathrm{OCH}_{3}$ \\
\hline $338.23441+$ & {$[\mathrm{M}+\mathrm{H}]^{+}-\mathrm{C}_{9} \mathrm{H}_{20} \mathrm{O}_{5} \mathrm{Si}$} \\
\hline 449.3385 & $\mathrm{M}-4\left(-\mathrm{OCH}_{3}\right)$ \\
\hline $475.35501+$ & {$[\mathrm{M}+\mathrm{Na}]^{+}-\mathrm{Si}(-\mathrm{OCH})_{3}$} \\
\hline 486.2541 & $\mathrm{M}-\mathrm{CH}_{3} \mathrm{OH}-\mathrm{C}_{4} \mathrm{H}_{8}$ \\
\hline $518.27951+$ & {$[\mathrm{M}+\mathrm{H}]^{+}-\mathrm{C}_{4} \mathrm{H}_{8}$} \\
\hline $542.31631+$ & {$[\mathrm{M}+\mathrm{H}]^{+}-\mathrm{CH}_{3} \mathrm{OH}$} \\
\hline $574.34361+$ & {$[\mathrm{M}+\mathrm{H}]^{+}$} \\
\hline $596.32371+$ & {$[\mathrm{M}+\mathrm{Na}]^{+}$} \\
\hline
\end{tabular}


Table S3. The mass-to-charge ratio $(\mathrm{m} / \mathrm{z})$ and its corresponding fragment of the molecular ion peaks of OG- BSQ.

\begin{tabular}{|c|c|}
\hline $\mathrm{m} / \mathrm{z}$ & Fragment \\
\hline 130.1504 & $\mathrm{M}-2 \mathrm{C}_{9} \mathrm{H}_{20} \mathrm{O}_{5} \mathrm{Si}$ \\
\hline $334.21971+$ & {$[\mathrm{M}+\mathrm{H}]^{+}-\mathrm{C}_{9} \mathrm{H}_{20} \mathrm{O}_{5} \mathrm{Si}-\mathrm{OCH}_{3}$} \\
\hline $366.24561+$ & {$[\mathrm{M}+\mathrm{H}]^{+}-\mathrm{C}_{9} \mathrm{H}_{20} \mathrm{O}_{5} \mathrm{Si}$} \\
\hline $486.22591+$ & {$[\mathrm{M}+\mathrm{H}]^{+}-\mathrm{CH}_{3} \mathrm{OH}-\mathrm{C}_{6} \mathrm{H}_{12}$} \\
\hline $518.25091+$ & {$[\mathrm{M}+\mathrm{H}]^{+}-\mathrm{C}_{6} \mathrm{H}_{12}$} \\
\hline 538.2908 & $\mathrm{M}-\mathrm{CH}_{3} \mathrm{OH}-\mathrm{OCH}_{3}$ \\
\hline $570.31761+$ & {$[\mathrm{M}+\mathrm{H}]^{+}-\mathrm{CH}_{3} \mathrm{OH}$} \\
\hline $592.29621+$ & {$[\mathrm{M}+\mathrm{Na}]^{+}-\mathrm{CH}_{3} \mathrm{OH}$} \\
\hline $602.34311+$ & {$[\mathrm{M}+\mathrm{H}]^{+}$} \\
\hline $624.32121+$ & {$[\mathrm{M}+\mathrm{Na}]^{+}$} \\
\hline
\end{tabular}




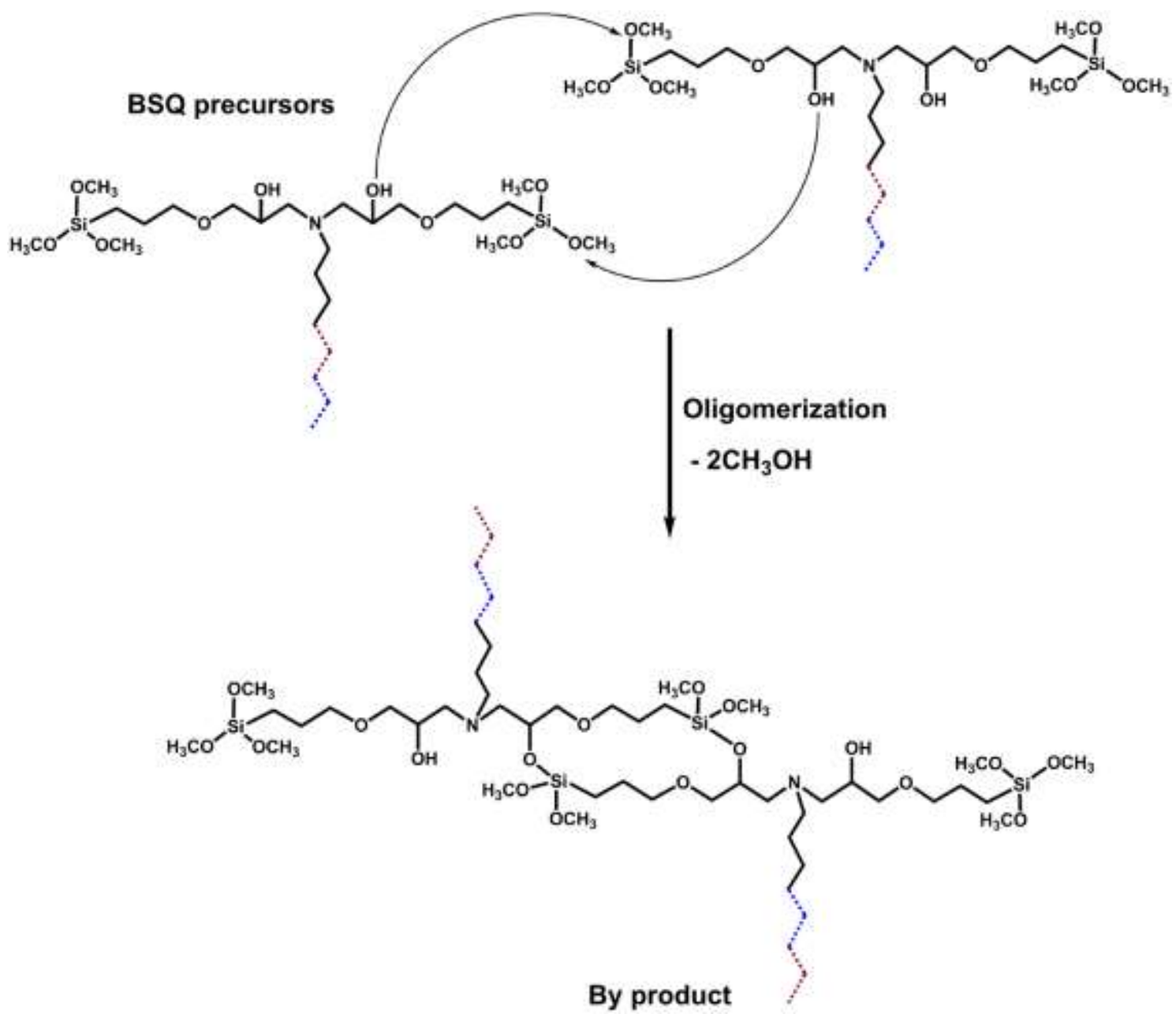

Figure S4. Side-reaction scheme of the formation of the by-product. 
(a) $\mathrm{m} / \mathrm{z}=1027.60731+$
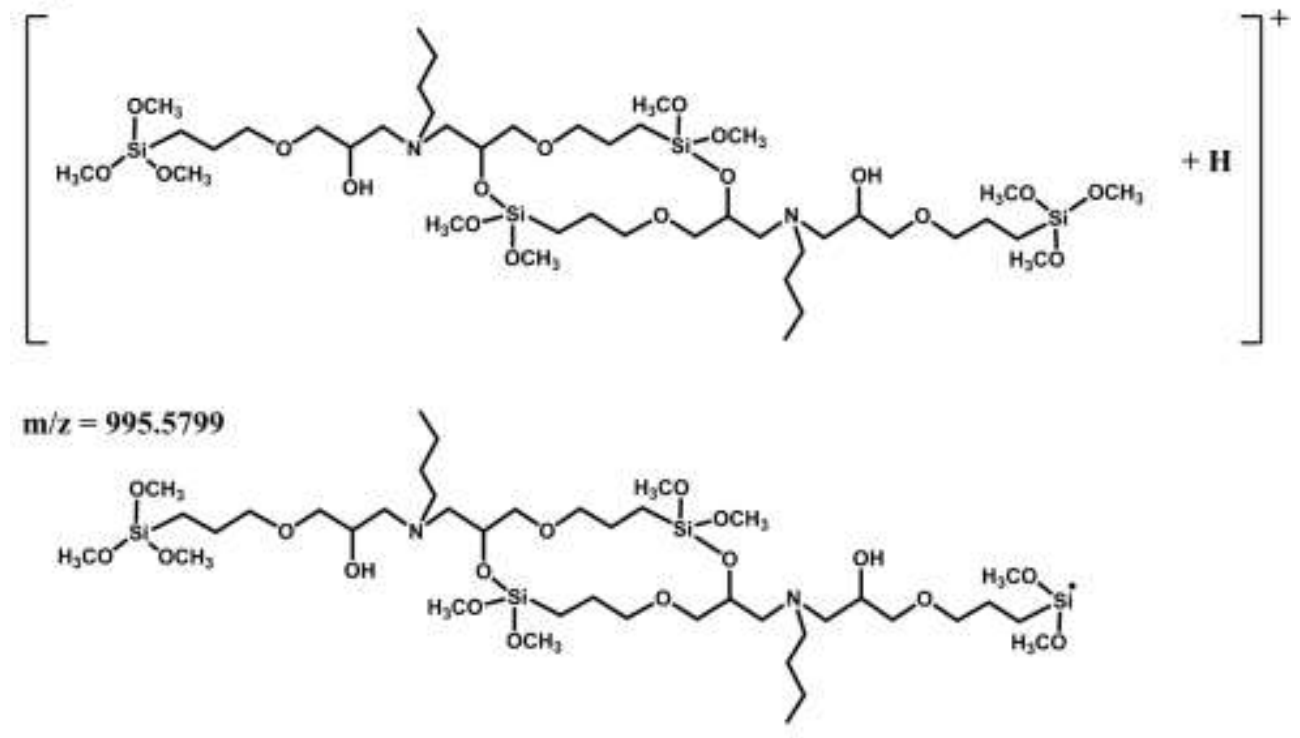

$\mathrm{m} / \mathrm{z}=755.4494$

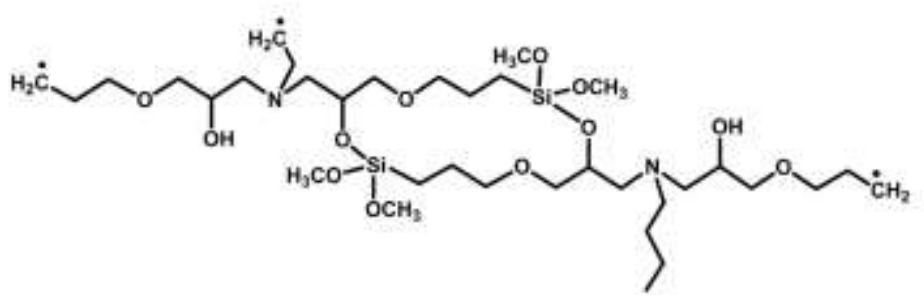



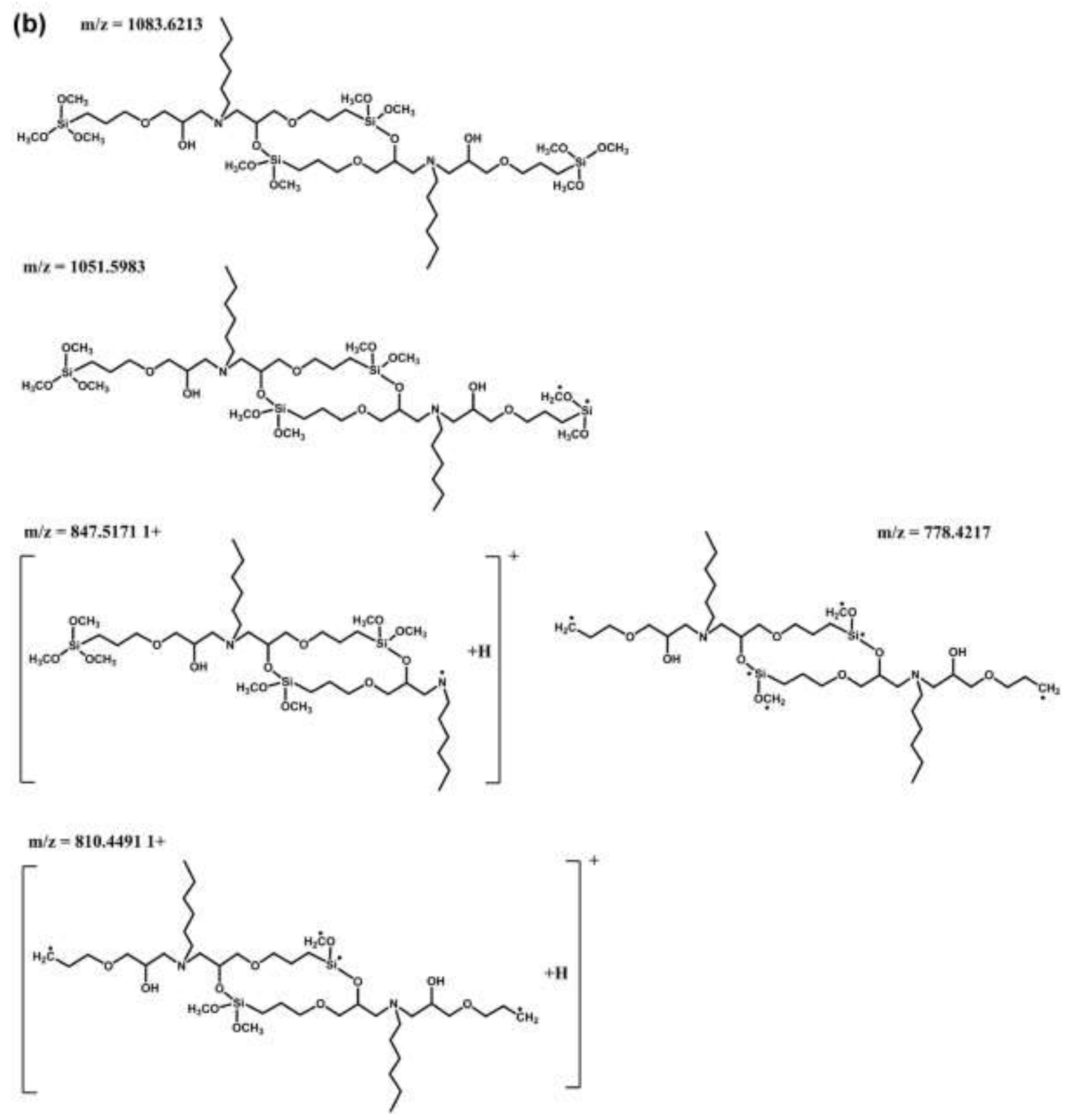

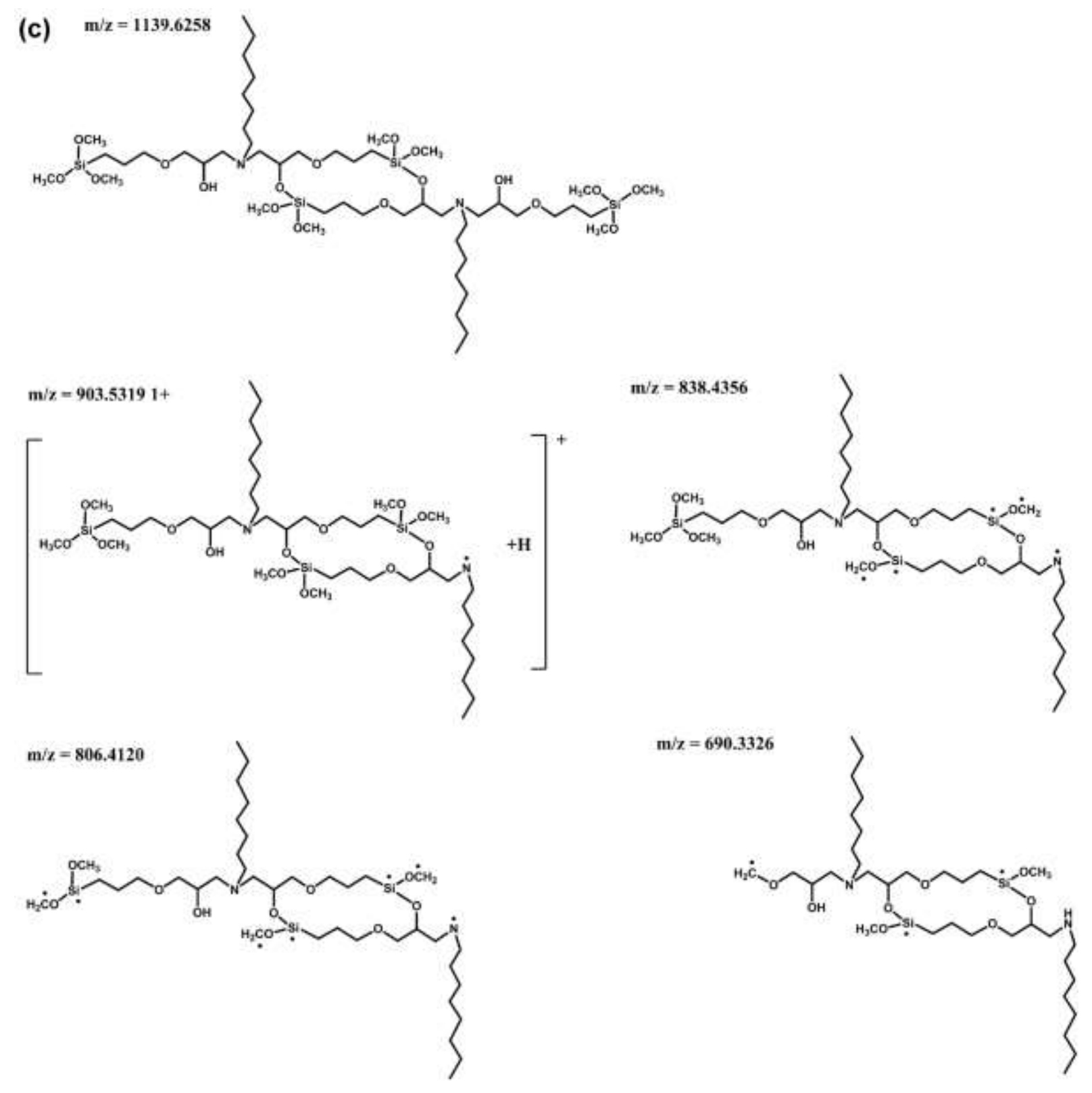

Figure S5. The mass-to-charge ratio $(\mathrm{m} / \mathrm{z})$ and its corresponding fragment structure of the molecular ion peaks of the by-product from (a) BG-BSQ, (b) HG-BSQ and (c) OG-BSQ. 


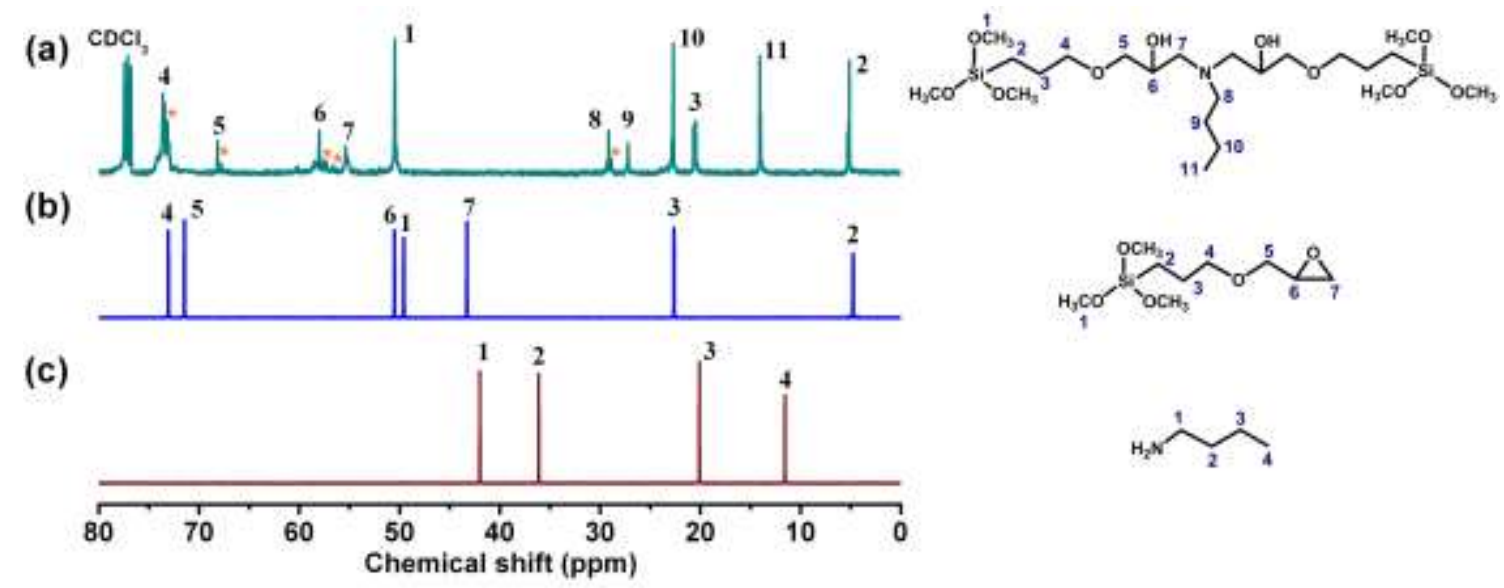

Figure S6. ${ }^{13} \mathrm{C}$ liquid NMR spectra of (a) BG-BSQ, (b) GPTMS and (c) BA.

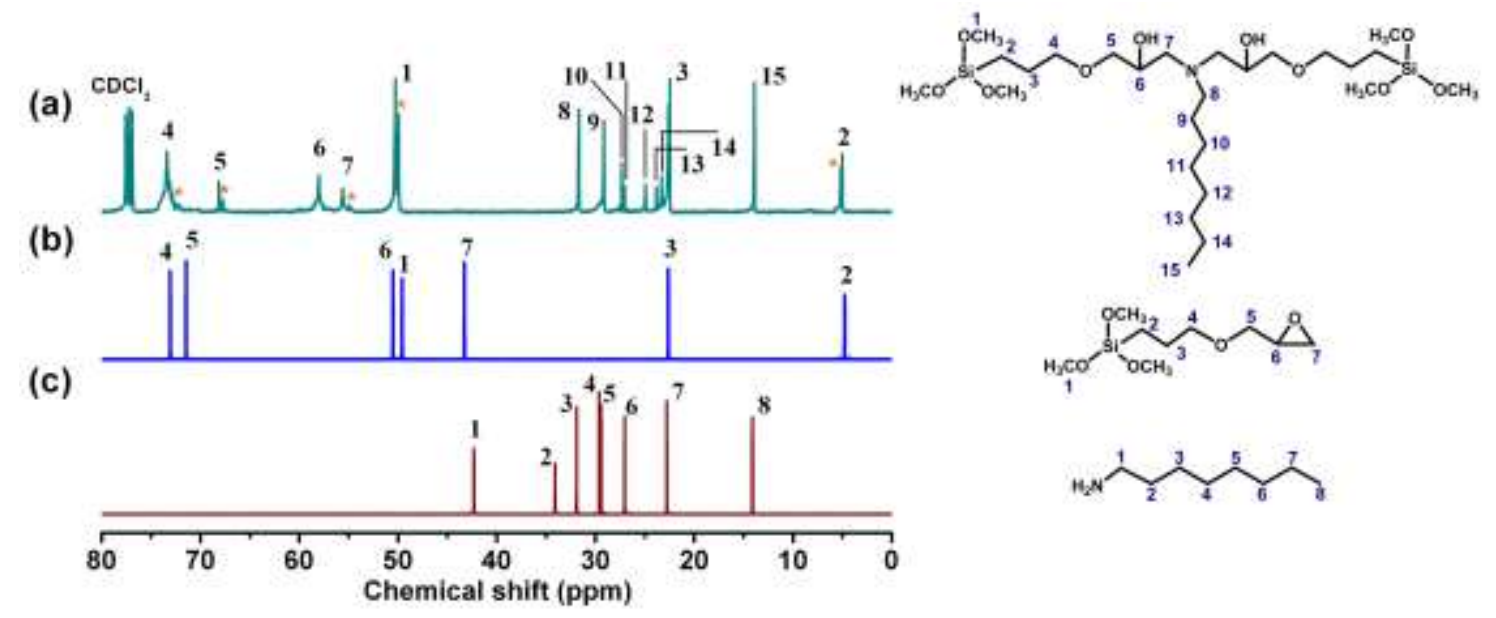

Figure S7. ${ }^{13} \mathrm{C}$ liquid NMR spectra of (a) OG-BSQ, (b) GPTMS and (c) OA. 


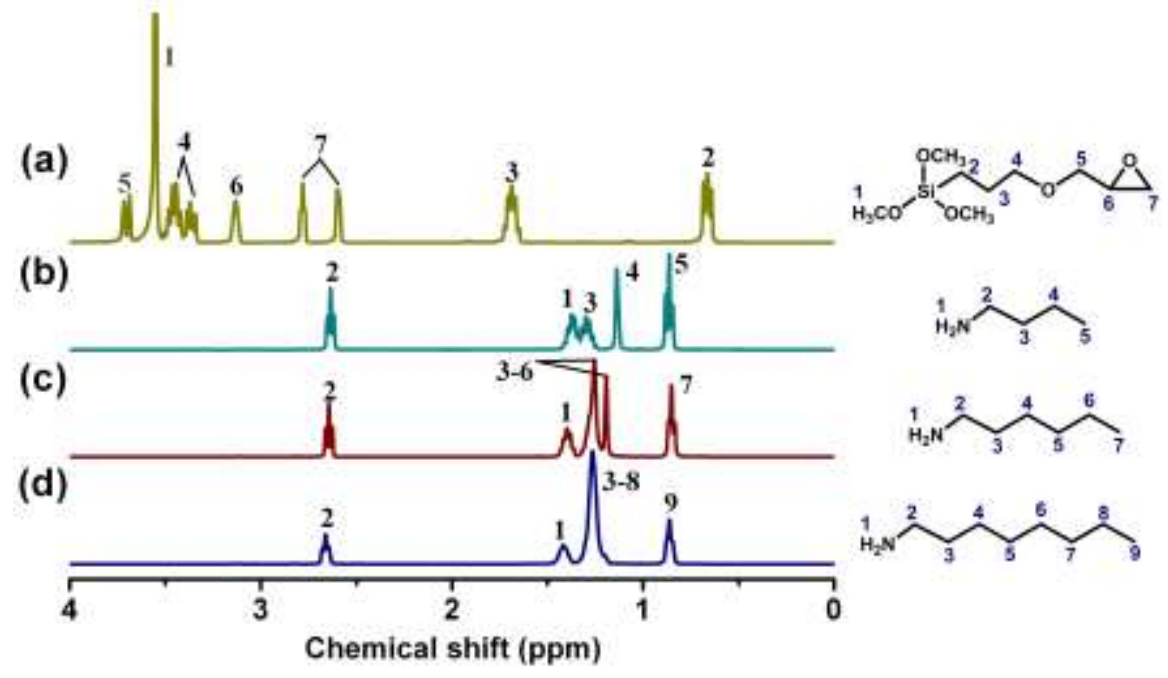

Figure S8. ${ }^{1} \mathrm{H}$ liquid NMR spectra of (a) GPMTS, (b) BA, (c) HA and (d) OA.

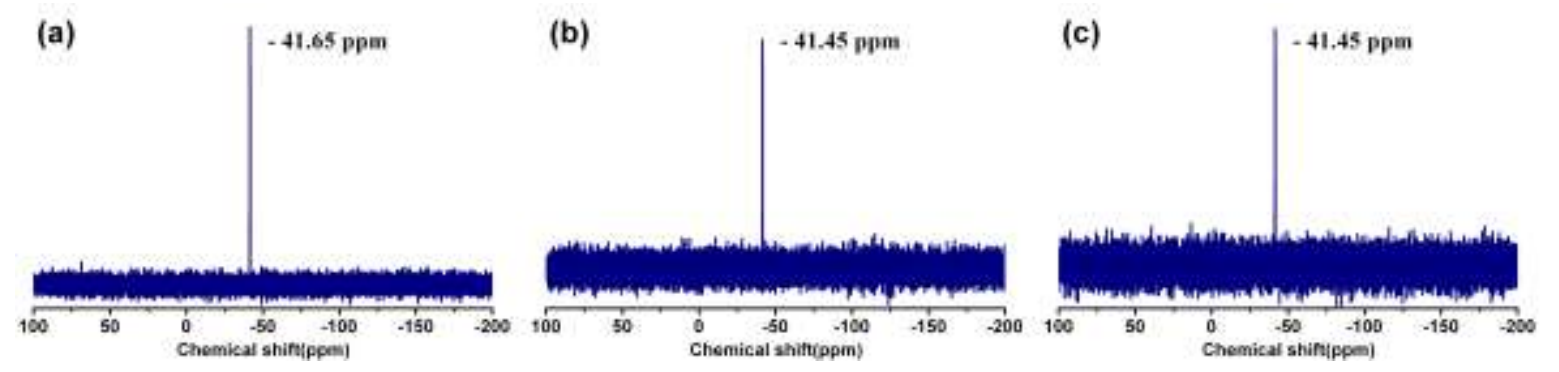

Figure S9. ${ }^{29}$ Si liquid NMR spectra of (a) BG-BSQ, (b) HG-BSQ and (c) OG-BSQ.
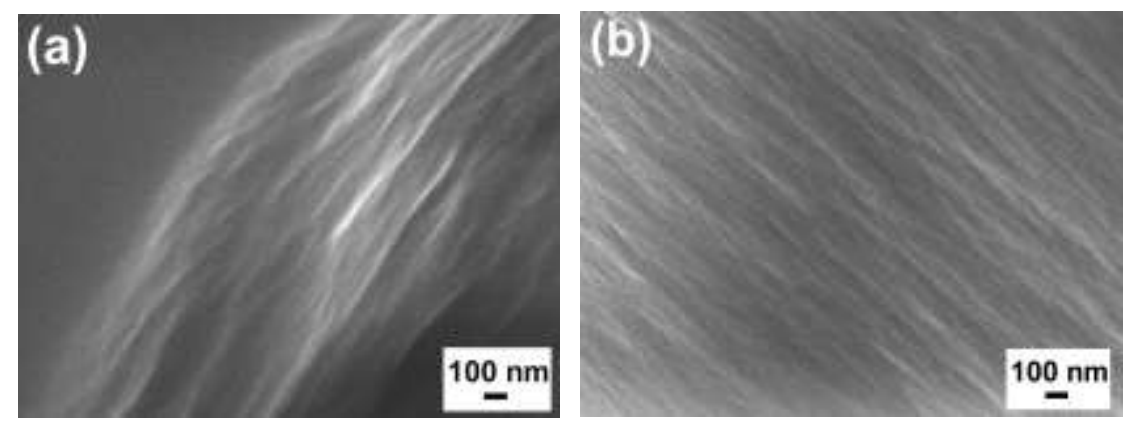

Figure S10. SEM images of the oblique-section of (a) F-BG and (b) F-OG. 

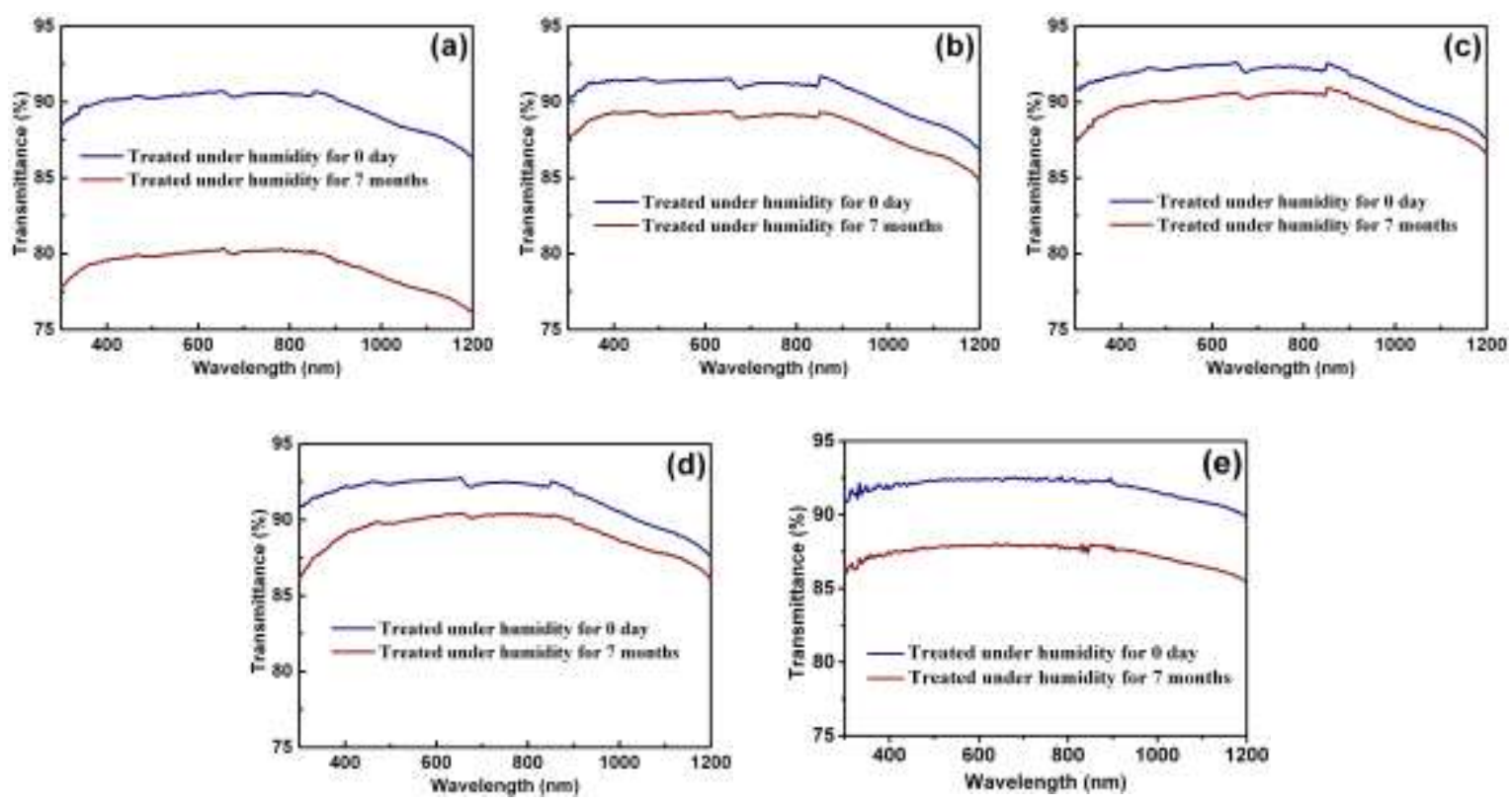

Figure S11. Transmittance of (a) bare DKDP and DKDP coated with (b) F-BG, (c) F-HG, (d) F$\mathrm{OG}$, (e) F-IG before and after treated under $60 \% \mathrm{RH}$ at $25^{\circ} \mathrm{C}$ for 7 months.
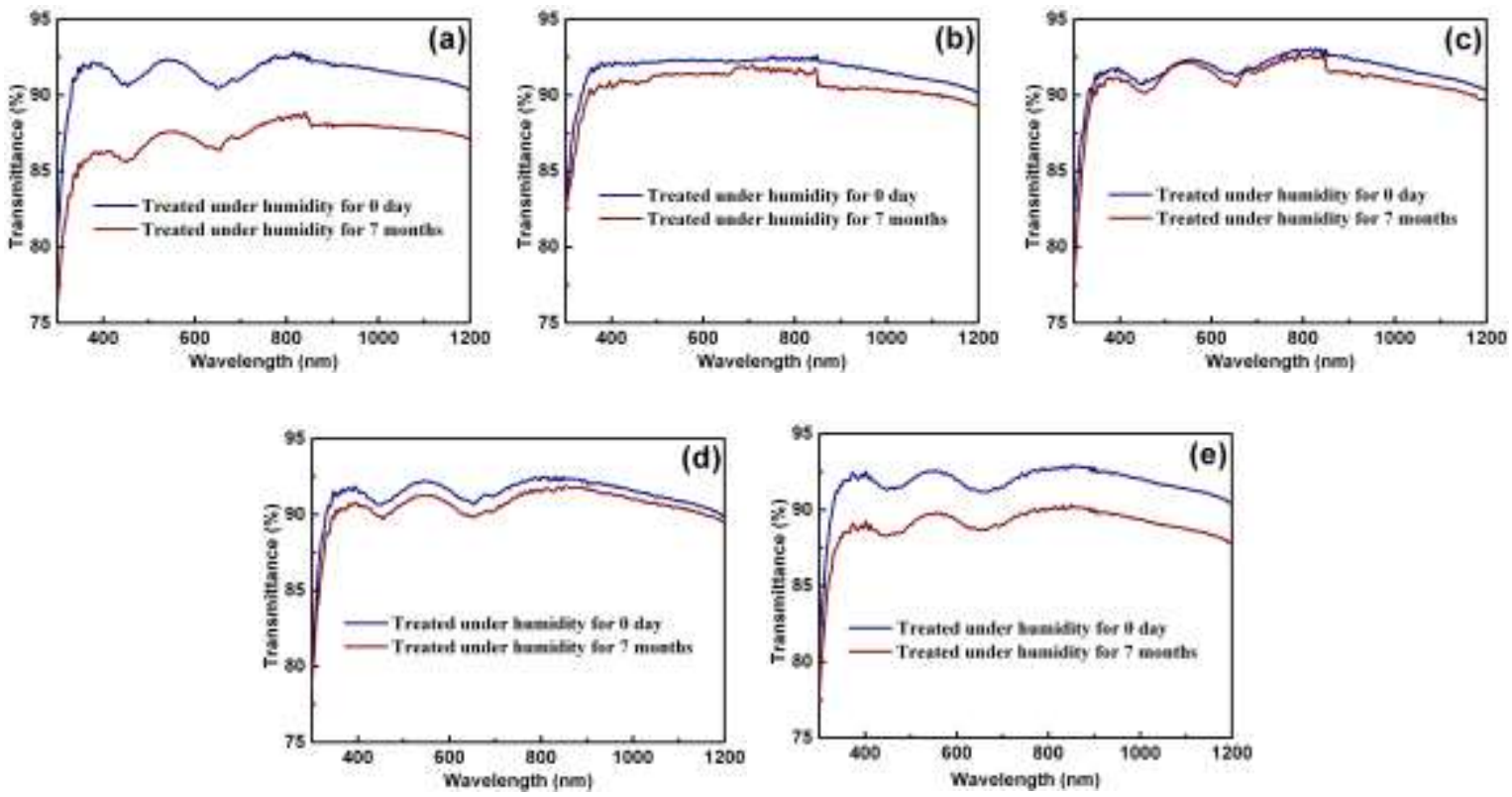

Figure S12. Transmittance of (a) bare KDP and KDP coated with (b) F-BG, (c) F-HG, (d) F-OG, (e) F-IG before and after treated under $60 \% \mathrm{RH}$ at $25^{\circ} \mathrm{C}$ for 7 months. 


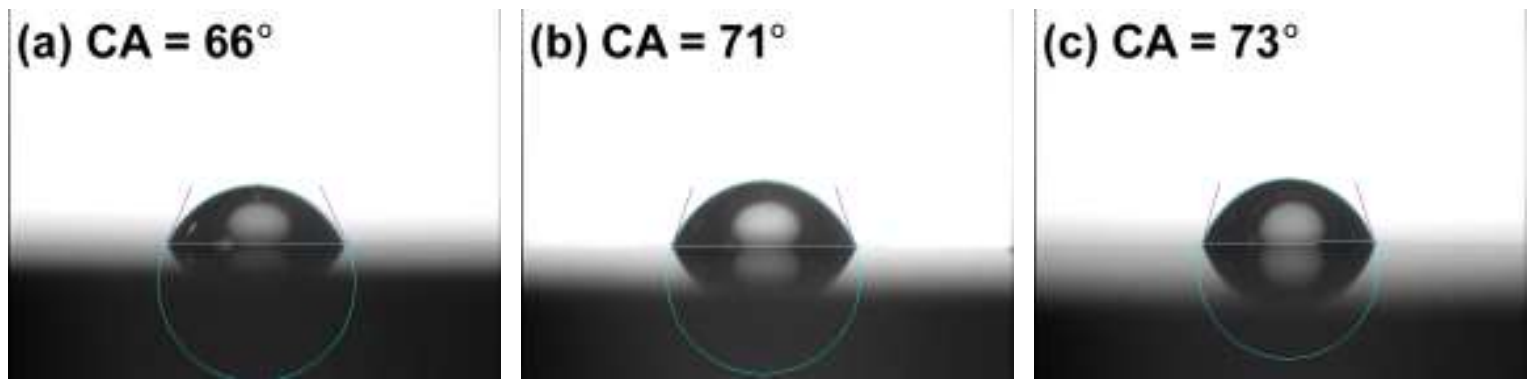

Figure S13. Water contact angle (CA) of three BPSQ films (a) F-BG, (b) F-HG and (c) F-OG. 